\title{
EXISTENCE AND EXPONENTIAL STABILITY OF ALMOST PERIODIC SOLUTIONS FOR CELLULAR NEURAL NETWORKS WITHOUT GLOBAL LIPSCHITZ CONDITIONS
}

\author{
BingWEN LIU
}

\begin{abstract}
In this paper cellular neutral networks with time-varying delays and continuously distributed delays are considered. Without assuming the global Lipschitz conditions of activation functions, some sufficient conditions for the existence and exponential stability of the almost periodic solutions are established by using the fixed point theorem and differential inequality techniques. The results of this paper are new and complement previously known results.
\end{abstract}

\section{Introduction}

Consider the following models for cellular neural networks (CNNs) with time-varying delays and continuously distributed delays

$$
\begin{aligned}
x_{i}^{\prime}(t)= & -c_{i}(t) x_{i}(t)+\sum_{j=1}^{n} a_{i j}(t) \tilde{g}_{j}\left(x_{j}\left(t-\tau_{i j}(t)\right)\right) \\
& +\sum_{j=1}^{n} b_{i j}(t) \int_{0}^{\infty} K_{i j}(u) g_{j}\left(x_{j}(t-u)\right) d u+I_{i}(t), \quad i=1,2, \ldots, n,
\end{aligned}
$$

which $n$ corresponds to the number of units in a neural network, $x_{i}(t)$ corresponds to the state vector of the $i$ th unit at the time $t, c_{i}(t)>0$ represents the rate with which the $i$ th unit will reset its potential to the resting state in isolation when disconnected from the network and external inputs at the time $t . a_{i j}(t)$ and $b_{i j}(t)$ are the connection weights at the time $t, \tau_{i j}(t) \geq 0$ corresponds to the transmission delay of the $i$ th unit along the axon of the $j$ th unit at the time $t$, and $I_{i}(t)$ denote the external inputs at time $t . \tilde{g}_{j}$ and $g_{j}$ are activation functions of signal transmission.

Received February 6, 2006.

2000 Mathematics Subject Classification. 34C25, 34K13.

Key words and phrases. cellular neural networks, almost periodic solution, exponential stability, fixed point theorem, delays.

This work was supported by the NNSF (10371034) of China, the Doctor Program Foundation of the Ministry of Education of China (20010532002) and Key Project of Chinese Ministry of Education ([2002]78). 
It is well known that the CNNs have been successfully applied to signal and image processing, pattern recognition and optimization. Hence, they have been the object of intensive analysis by numerous authors in recent years. In particular, there have been extensive results on the problem of the existence and stability of periodic and almost periodic solutions of CNNs in the literature. We refer the reader to $[2-6,10-15]$ and the references cited therein. Moreover, in the above-mentioned literature, we observe that the following assumption

$\left(H_{0}\right)$ for each $j \in\{1,2, \ldots, n\}, \tilde{g}_{j}, g_{j}: R \rightarrow R$ are global Lipschitz with Lipschitz constants $\tilde{L}_{j}$ and $L_{j}$, i.e.,

$$
\left|\tilde{g}_{j}(u)-\tilde{g}_{j}(v)\right| \leq \tilde{L}_{j}|u-v|,\left|g_{j}(u)-g_{j}(v)\right| \leq L_{j}|u-v| \text { for all } u, v \in R \text {. }
$$

has been considered as fundamental for the considered existence and stability of periodic and almost periodic solutions of CNNs. However, to the best of our knowledge, few authors have considered the problems of almost periodic solutions of CNNs without the assumptions $\left(H_{0}\right)$. Thus, it is worth while to continue to investigate the existence and stability of almost periodic solutions of CNNs in this case.

The main purpose of this paper is to give the conditions for the existence and exponential stability of the almost periodic solutions for system (1.1). By applying fixed point theorem and differential inequality techniques, we derive some new sufficient conditions ensuring the existence, uniqueness and exponential stability of the almost periodic solution, which are new and they complement previously known results. In particular, we do not need the assumption $\left(H_{0}\right)$. Moreover, an example is also provided to illustrate the effectiveness of our results.

Throughout this paper, for $i, j=1,2, \ldots, n$, it will be assumed that $c_{i}, I_{i}, a_{i j}$, $b_{i j}, \tau_{i j}: R \rightarrow R$ are almost periodic functions, and there exist constants $\tau, \tilde{c}_{i}, \overline{a_{i j}}, \overline{b_{i j}}$ and $\overline{I_{i}}$ such that

$$
\begin{aligned}
\tau & =\max _{1 \leq i, j \leq n} \sup _{t \in R} \tau_{i j}(t), 0<\tilde{c}_{i}=\inf _{t \in R} c_{i}(t), \\
\sup _{t \in R}\left|b_{i j}(t)\right| & =\overline{b_{i j}}, \sup _{t \in R}\left|a_{i j}(t)\right|=\overline{a_{i j}} \sup _{t \in R}\left|I_{i}(t)\right|=\overline{I_{i}} .
\end{aligned}
$$
hold.

We also assume that the following conditions $\left(H_{1}\right),\left(H_{2}\right),\left(H_{3}\right)$ and $\left(H_{4}\right)$

$\left(H_{1}\right)$ For each $j \in\{1,2, \ldots, n\}$, there exist $\tilde{f}_{j}, \tilde{h}_{j}, f_{j}, h_{j} \in C(R, R)$ and constants $L_{j}^{\tilde{f}}, L_{j}^{\tilde{h}}, \tilde{M}_{j}, L_{j}^{f}, L_{j}^{h}, M_{j} \in[0,+\infty)$ such that the following conditions are satisfied.

(1) $\tilde{f}_{j}(0)=0, \tilde{h}_{j}(0)=0, \tilde{g}_{j}(u)=\tilde{f}_{j}(u) \tilde{h}_{j}(u),\left|\tilde{h}_{j}(u)\right| \leq \tilde{M}_{j}$ for all $u \epsilon$ $R$;

(2) $f_{j}(0)=0, h_{j}(0)=0, g_{j}(u)=f_{j}(u) h_{j}(u),\left|h_{j}(u)\right| \leq M_{j}$ for all $u \in$ $R$;

(3) $\left|\tilde{f}_{j}(u)-\tilde{f}_{j}(v)\right| \leq L_{j}^{\tilde{f}}|u-v|,\left|\tilde{h}_{j}(u)-\tilde{h}_{j}(v)\right| \leq L_{j}^{\tilde{h}}|u-v|$ for all $u, v \in$ $R$; 
(4) $\left|f_{j}(u)-f_{j}(v)\right| \leq L_{j}^{f}|u-v|,\left|h_{j}(u)-h_{j}(v)\right| \leq L^{h}|u-v|$ for all $u, v \in$ $R$.

$\left(H_{2}\right)$ For $i, j \in\{1,2, \ldots, n\}$, the delay kernels $K_{i j}:\{0, \infty) \rightarrow R$ are continuous, integrable and satisfy

$$
\int_{0}^{\infty}\left|K_{i j}(s)\right| d s \leq k_{i j}
$$

$\left(H_{3}\right)$ Assume that there exist nonnegative constants $L, d_{i j}$ and $\delta$ such that

$$
\begin{gathered}
L=\max _{1 \leq i \leq n}\left\{\frac{\overline{I_{i}}}{\tilde{\tilde{c}_{i}}}\right\}, \delta=\max _{1 \leq i \leq n}\left\{\sum_{j=1}^{n} \tilde{c}_{i}^{-1}\left[\overline{a_{i j}} L_{j}^{\tilde{f}} \tilde{M}_{j}+\widetilde{b_{i j}} k_{i j} L_{j}^{f} M_{j}\right]\right\}<1, \\
d_{i j}=\tilde{c}_{i}^{-1}\left\langle\overline{a_{i j}} L_{j}^{\tilde{f}}\left(L_{j}^{\hat{h}} \frac{L}{1-\delta}+\tilde{M}_{j}\right)+\overline{b_{i j}} k_{i j} L_{j}^{f}\left(L_{j}^{h} \frac{L}{1-\delta}+M_{j}\right)\right), i, j=1,2, \ldots, n .
\end{gathered}
$$

$\left(H_{4}\right)$ For $i, j \in\{1,2, \ldots, n\}$, there exists a constant $\lambda_{0}>0$ such that

$$
\int_{0}^{\infty}\left|K_{i j}(s)\right| e^{\lambda_{0} s} d s<+\infty
$$

For convenience, we introduce some notations. We will use $x=\left(x_{1}, x_{2}, \ldots\right.$, $\left.x_{n}\right)^{T} \in R^{n}$ to denote a column vector, in which the symbol $\left({ }^{T}\right)$ denotes the transpose of a vector. For matrix $D=\left(d_{i j}\right)_{n \times n}, D^{T}$ denotes the transpose of $D$, and $E_{n}$ denotes the identity matrix of size $n$. A matrix or vector $D \geq 0$ means that all entries of $D$ are greater than or equal to zero. $D>0$ can be defined similarly. For matrices or vectors $D$ and $E, D \geq E$ (resp. $D>E$ ) means that $D-E \geq 0$ (resp. $D-E>0$ ).

Throughout this paper, we set

$$
\begin{aligned}
\left\{x_{j}(t)\right\} & =\left(x_{1}(t), x_{2}(t), \ldots, x_{n}(t)\right)^{T} \text { and } \\
B & =\left\{\varphi \mid \varphi=\left\{\varphi_{j}(t)\right\}=\left(\varphi_{1}(t), \varphi_{2}(t), \ldots, \varphi_{n}(t)\right)^{T}\right\},
\end{aligned}
$$

where $\varphi$ is an almost periodic function on $R$. For $\forall \varphi \in B$, we define induced module $\|\varphi\|_{B}=\sup _{t \in R}\|\varphi(t)\|$, then $B$ is a Banach space.

The initial conditions associated with system (1.1) are of the form

$$
x_{i}(s)=\varphi_{i}(s), s \in(-\infty, 0], i=1,2, \ldots, n,
$$

where $\varphi_{i}(\cdot)$ denotes real-valued bounded continuous function defined on $(-\infty$, $0]$.

Definition 1 ([see 7, 9]). Let $u(t): R \longrightarrow R^{n}$ be continuous in $t . u(t)$ is said to be almost periodic on $R$ if, for any $\varepsilon>0$, the set $T(u, \varepsilon)=\{\delta:|u(t+\delta)-u(t)|<$ $\varepsilon, \forall t \in R\}$ is relatively dense, i.e., for $\forall \varepsilon>0$, it is possible to find a real number $l=l(\varepsilon)>0$, for any interval with length $l(\varepsilon)$, there exists a number $\delta=\delta(\varepsilon)$ in this interval such that $|u(t+\delta)-u(t)|<\varepsilon$, for $\forall t \in R$. 
Definition 2. Let $Z^{*}(t)=\left(x_{1}^{*}(t), x_{2}^{*}(t), \ldots, x_{n}^{*}(t)\right)^{T}$ be an almost periodic solution of system (1.1) with initial value $\varphi^{*}=\left(\varphi_{1}^{*}(t), \varphi_{2}^{*}(t), \ldots, \varphi_{n}^{*}(t)\right)^{T}$. If there exist constants $\lambda>0$ and $M_{\varphi}>1$ such that for every solution $Z(t)=\left(x_{1}(t), x_{2}(t), \ldots, x_{n}(t)\right)^{T}$ of system $(1.1)$ with any initial value $\varphi=$ $\left(\varphi_{1}(t), \varphi_{2}(t), \ldots, \varphi_{n}(t)\right)^{T}$

$$
\left|x_{i}(t)-x_{i}^{*}(t)\right| \leq M_{\varphi}\left\|\varphi-\varphi^{*}\right\|_{1} e^{-\lambda t}, \forall t>0, \quad i=1,2, \ldots, n,
$$

where $\left\|\varphi-\varphi^{*}\right\|_{1}=\sup _{-\infty \leq s \leq 0} \max _{1 \leq i \leq n}\left|\varphi_{i}(s)-\varphi_{i}^{*}(s)\right|$. Then $Z^{*}(t)$ is said to be global exponential stable.

Definition 3 ([see 7,9]). Let $x \in R^{n}$ and $Q(t)$ be a $n \times n$ continuous matrix defined on $R$. The linear system

$$
x^{\prime}(t)=Q(t) x(t)
$$

is said to admit an exponential dichotomy on $R$ if there exist positive constants $k, \alpha$, projection $P$ and the fundamental solution matrix $X(t)$ of (1.5) satisfying

$$
\begin{aligned}
& \left\|X(t) P X^{-1}(s)\right\| \leq k e^{-\alpha(t-s)} \text { for } t \geq s, \\
& \left\|X(t)(I-P) X^{-1}(s)\right\| \leq k e^{-\alpha(s-t)} \quad \text { for } t \leq s .
\end{aligned}
$$

Lemma 1.1 ([see 7,9]). If the linear system (1.5) admits an exponential dichotomy, then almost periodic system

$$
x^{\prime}(t)=Q(t) x+g(t)
$$

has a unique almost periodic solution $x(t)$, and

$$
x(t)=\int_{-\infty}^{t} X(t) P X^{-1}(s) g(s) d s-\int_{t}^{+\infty} X(t)(I-P) X^{-1}(s) g(s) d s .
$$

Lemma 1.2 ([see 7, 9]). Let $c_{i}(t)$ be an almost periodic function on $R$ and

$$
M\left[c_{i}\right]=\lim _{T \rightarrow+\infty} \frac{1}{T} \int_{t}^{t+T} c_{i}(s) d s>0, \quad i=1,2, \ldots, n .
$$

Then the linear system

$$
x^{\prime}(t)=\operatorname{diag}\left(-c_{1}(t),-c_{2}(t), \ldots,-c_{n}(t)\right) x(t)
$$

admits an exponential dichotomy on $R$.

Definition 4 ([see 1]). A real $n \times n$ matrix $W=\left(w_{i j}\right)_{n \times n}$ is said to be an $M$ matrix if $w_{i j} \leq 0, i, j=1,2, \ldots, n, i \neq j$, and $W^{-1} \geq 0$, where $W^{-1}$ denotes the inverse of $W$.

Lemma 1.3 ([see 1]). Let $W=\left(w_{i j}\right)_{n \times n}$ with $w_{i j} \leq 0, i, j=1,2, \ldots, n, i \neq j$. Then the following statements are equivalent.

(1) $W$ is an $M$-matrix. 0 .

(2) There exists a vector $\eta=\left(\eta_{1}, \eta_{2}, \ldots, \eta_{n}\right)>(0,0, \ldots, 0)$ such that $\eta W>$

(3) There exists a vector $\xi=\left(\xi_{1}, \xi_{2}, \ldots, \xi_{n}\right)^{T}>(0,0, \ldots, 0)^{T}$ such that $W \xi>0$. 
Lemma 1.4 ([see 1]). Let $A \geq 0$ be an $n \times n$ matrix and $\rho(A)<1$, then $\left(E_{n}-A\right)^{-1} \geq 0$, where $\rho(A)$ denotes the spectral radius of $A$.

The remaining part of this paper is organized as follows. In Section 2, we shall derive new sufficient conditions for checking the existence of almost periodic solutions of (1.1). In Section 3, we present some new sufficient conditions for the uniqueness and exponential stability of the almost periodic solution of (1.1). In Section 4, we shall give some examples and remarks to illustrate our results obtained in the previous sections.

\section{Existence of almost periodic solutions}

Theorem 2.1. Let $\rho(D)=\rho\left(\left(d_{i j}\right)_{n \times n}\right)<1$. Suppose that the conditions $\left(H_{1}\right)$, $\left(H_{2}\right)$ and $\left(H_{3}\right)$ hold. Then, there exists a unique almost periodic solution of system (1.1) in the region $B^{*}=\left\{\varphi \mid \varphi \in B,\left\|\varphi-\varphi_{0}\right\|_{B} \leq \frac{\delta L}{1-\delta}\right\}$, where

$$
\begin{aligned}
\varphi_{0}(t)= & \left\{\int_{-\infty}^{t} e^{-\int_{s}^{t} c_{j}(u) d u} I_{j}(s) d s\right\} \\
= & \left(\int_{-\infty}^{t} e^{-\int_{s}^{t} c_{1}(u) d u} I_{1}(s) d s\right. \\
& \left.\int_{-\infty}^{t} e^{-\int_{s}^{t} c_{2}(u) d u} I_{2}(s) d s, \ldots, \int_{-\infty}^{t} e^{-\int_{s}^{t} c_{n}(u) d u} I_{n}(s) d s\right)^{T} .
\end{aligned}
$$

Proof. For $\forall \varphi \in B$, we consider the almost periodic solution $x^{\varphi}(t)$ of nonlinear almost periodic differential equations

$$
\begin{aligned}
x_{i}^{\prime}(t)= & -c_{i}(t) x_{i}(t)+\sum_{j=1}^{n} a_{i j}(t) \tilde{g}_{j}\left(\varphi_{j}\left(t-\tau_{i j}(t)\right)\right) \\
& +\sum_{j=1}^{n} b_{i j}(t) \int_{0}^{\infty} K_{i j}(u) g_{j}\left(\varphi_{j}(t-u)\right) d u+I_{i}(t), \quad i=1,2, \ldots, n .
\end{aligned}
$$

Then, notice that $M\left[c_{i}\right]>0, i=1,2, \ldots, n$, it follows from Lemma 1.2 that the linear system

$$
x^{\prime}(t)=-c_{i}(t) x(t), \quad i=1,2, \ldots, n,
$$

admits an exponential dichotomy on $R$. Thus, by Lemma 1.1, we obtain that the system (2.1) has exactly one almost periodic solution:

$$
\begin{aligned}
& x^{\varphi}(t) \\
= & \left(x_{1}^{\varphi}(t), x_{2}^{\varphi}(t), \ldots, x_{n}^{\varphi}(t)\right)^{T} \\
= & \left(\int _ { - \infty } ^ { t } e ^ { - \int _ { s } ^ { t } c _ { 1 } ( u ) d u } \left[\sum_{j=1}^{n} a_{1 j}(s) \tilde{g}_{j}\left(\varphi_{j}\left(s-\tau_{1 j}(s)\right)\right)+\sum_{j=1}^{n} b_{1 j}(s) \int_{0}^{\infty} K_{1 j}(u)\right.\right.
\end{aligned}
$$




$$
\begin{aligned}
& \left.\cdot g_{j}\left(\varphi_{j}(s-u)\right) d u+I_{1}(s)\right] d s, \ldots, \\
& \int_{-\infty}^{t} e^{-\int_{s}^{t} c_{n}(u) d u}\left[\sum_{j=1}^{n} a_{n j}(s) \tilde{g}_{j}\left(\varphi_{j}\left(s-\tau_{n j}(s)\right)\right)\right. \\
& \left.\left.+\sum_{j=1}^{n} b_{n j}(s) \int_{0}^{\infty} K_{n j}(u) g_{j}\left(\varphi_{j}(s-u)\right) d u+I_{n}(s)\right] d s\right)^{T} .
\end{aligned}
$$

Now, we define a mapping $T: B \rightarrow B$ by setting

$$
T(\varphi)(t)=x^{\varphi}(t), \quad \forall \varphi \in B
$$

Since $B^{*}=\left\{\varphi \mid \varphi \in B,\left\|\varphi-\varphi_{0}\right\|_{B} \leq \frac{\delta L}{1-\delta}\right\}$, it is easy to see that $B^{*}$ is a closed convex subset of $B$. According to the definition of the norm of Banach space $B$, we get

$$
\begin{aligned}
\left\|\varphi_{0}\right\|_{B} & =\sup _{t \in R} \max _{1 \leq i \leq n}\left\{\int_{-\infty}^{t} I_{i}(s) e^{-\int_{s}^{t} c_{i}(u) d u} d s\right\} \\
& \leq \sup _{t \in R} \max _{1 \leq i \leq n}\left\{\frac{\overline{I_{i}}}{\tilde{c}_{i}}\right\}=\max _{1 \leq i \leq n}\left\{\frac{\overline{I_{i}}}{\tilde{c}_{i}}\right\}=L .
\end{aligned}
$$

Therefore, for $\forall \varphi \in B^{*}$, we have

$$
\|\varphi\|_{B} \leq\left\|\varphi-\varphi_{0}\right\|_{B}+\left\|\varphi_{0}\right\|_{B} \leq \frac{\delta L}{1-\delta}+L=\frac{L}{1-\delta} .
$$

For $j=1,2, \ldots, n$, in view of $\left(H_{1}\right)$, we have

$$
\begin{aligned}
& \left|\tilde{f}_{j}(u)\right| \leq L_{j}^{\tilde{f}}|u|,\left|\tilde{h}_{j}(u)\right| \leq L_{j}^{\tilde{h}}|u|,\left|\tilde{h}_{j}(u)\right| \leq \tilde{M}_{j}, \\
& \left|f_{j}(u)\right| \leq L_{j}^{f}|u|,\left|h_{j}(u)\right| \leq L_{j}^{h}|u|,\left|h_{j}(u)\right| \leq M_{j}, \forall u \in R .
\end{aligned}
$$

Now, we prove that the mapping $T$ is a self-mapping from $B^{*}$ to $B^{*}$. In fact, for $\forall \varphi \in B^{*}$, together with (2.5) and (2.6), we obtain

$$
\begin{aligned}
& \left\|T \varphi-\varphi_{0}\right\|_{B} \\
= & \sup _{t \in R} \max _{1 \leq i \leq n}\left\{\mid \int_{-\infty}^{t} e^{-\int_{s}^{t} c_{i}(u) d u}\left[\sum_{j=1}^{n} a_{i j}(s) \tilde{g}_{j}\left(\varphi_{j}\left(s-\tau_{i j}(s)\right)\right)\right.\right. \\
& \left.\left.+\sum_{j=1}^{n} b_{i j}(s) \int_{0}^{\infty} K_{i j}(u) g_{j}\left(\varphi_{j}(s-u)\right) d u\right] d s \mid\right\} \\
\leq & \sup _{t \in R} \max _{1 \leq i \leq n}\left\{\int _ { - \infty } ^ { t } e ^ { - \int _ { s } ^ { t } c _ { i } ( u ) d u } \left[\sum_{j=1}^{n}\left|a_{i j}(s) \| \tilde{f}_{j}\left(\varphi_{j}\left(s-\tau_{i j}(s)\right)\right)\right|\left|\tilde{h}_{j}\left(\varphi_{j}\left(s-\tau_{i j}(s)\right)\right)\right|\right.\right. \\
& \left.\left.+\sum_{j=1}^{n}\left|b_{i j}(s)\right| \int_{0}^{\infty}\left|K_{i j}(u)\right|\left|f_{j}\left(\varphi_{j}(s-u)\right) \| h_{j}\left(\varphi_{j}(s-u)\right)\right| d u\right] d s\right\}
\end{aligned}
$$




$$
\begin{aligned}
& \leq \sup _{t \in R} \max _{1 \leq i \leq n}\left\{\int_{-\infty}^{t} e^{-\int_{s}^{t} c_{i}(u) d u} \sum_{j=1}^{n}\left[\overline{a_{i j}} L_{j}^{\tilde{f}} \tilde{M}_{j}+\overline{b_{i j}} k_{i j} L_{j}^{f} M_{j}\right] d s\|\varphi\|_{B}\right\} \\
& \leq \max _{1 \leq i \leq n}\left\{\sum_{j=1}^{n} \tilde{c}_{i}^{-1}\left[\overline{a_{i j}} L_{j}^{\tilde{f}} \tilde{M}_{j}+\overline{b_{i j}} k_{i j} L_{j}^{f} M_{j}\right]\right\}\|\varphi\|_{B} \\
& =\delta\|\varphi\|_{B} \leq \frac{\delta L}{1-\delta}
\end{aligned}
$$

where $\delta=\max _{1 \leq i \leq n}\left\{\sum_{j=1}^{n} \tilde{c}_{i}^{-1}\left[\overline{a_{i j}} L_{j}^{\tilde{f}} \tilde{M}_{j}+\overline{b_{i j}} k_{i j} L_{j}^{f} M_{j}\right]\right\}$, it implies that $T(\varphi)(t) \in B^{*}$ and $\|T \varphi\|_{B} \leq \frac{L}{1-\delta}$. So, the mapping $T$ is a self-mapping from $B^{*}$ to $B^{*}$. Hence, by using a similar argument of the proof of (2.7), we can obtain

$$
\left\|T^{m} \varphi-\varphi_{0}\right\|_{B} \leq \frac{\delta L}{1-\delta},\left\|T^{m} \varphi\right\|_{B} \leq \frac{L}{1-\delta},
$$

where $m$ is a positive integer, which implies that the mapping $T^{m}$ is a selfmapping from $B^{*}$ to $B^{*}$.

Next, we prove that there exists a positive integer $N$ such that the mapping $T^{N}$ is a contraction mapping of the $B^{*}$. In fact, in view of $(2.5),(2.6)$ and the condition $\left(H_{1}\right)$, for $\forall \phi, \psi \in B^{*}$, we have

$$
\begin{aligned}
& |T(\phi(t))-T(\psi(t))| \\
= & \left(\left|(T(\phi(t))-T(\psi(t)))_{1}\right|, \ldots,\left|(T(\phi(t))-T(\psi(t)))_{n}\right|\right)^{T} \\
= & \left(\mid \int_{-\infty}^{t} e^{-\int_{s}^{t} c_{1}(u) d u}\left[\sum_{j=1}^{n} a_{1 j}(s)\left(\tilde{g}_{j}\left(\phi_{j}\left(s-\tau_{1 j}(s)\right)\right)-\tilde{g}_{j}\left(\psi_{j}\left(s-\tau_{1 j}(s)\right)\right)\right)\right.\right. \\
& \left.+\sum_{j=1}^{n} b_{1 j}(s) \int_{0}^{\infty} K_{1 j}(u)\left(g_{j}\left(\phi_{j}(s-u)\right)-g_{j}\left(\psi_{j}(s-u)\right)\right) d u\right] d s \mid \\
& \ldots, \mid \int_{-\infty}^{t} e^{-\int_{s}^{t} c_{n}(u) d u}\left[\sum_{j=1}^{n} a_{n j}(s)\left(\tilde{g}_{j}\left(\phi_{j}\left(s-\tau_{n j}(s)\right)\right)-\tilde{g}_{j}\left(\psi_{j}\left(s-\tau_{n j}(s)\right)\right)\right)\right. \\
& \left.\left.+\sum_{j=1}^{n} b_{n j}(s) \int_{0}^{\infty} K_{n j}(u)\left(g_{j}\left(\phi_{j}(s-u)\right)-g_{j}\left(\psi_{j}(s-u)\right)\right) d u\right] d s \mid\right) T \\
\leq & \left(\int _ { - \infty } ^ { t } e ^ { - \int _ { s } ^ { t } c _ { 1 } ( u ) d u } \left[\sum _ { j = 1 } ^ { n } | a _ { 1 j } ( s ) | \left(\mid \tilde{f}_{j}\left(\phi_{j}\left(s-\tau_{1 j}(s)\right)\right) \tilde{h}_{j}\left(\phi_{j}\left(s-\tau_{1 j}(s)\right)\right)\right.\right.\right. \\
& -\tilde{f}_{j}\left(\phi_{j}\left(s-\tau_{1 j}(s)\right)\right) \tilde{h}_{j}\left(\psi_{j}\left(s-\tau_{1 j}(s)\right)\right)|+| \tilde{f}_{j}\left(\phi_{j}\left(s-\tau_{1 j}(s)\right)\right) \\
& \left.+\tilde{h}_{j}\left(\psi_{j}\left(s-\tau_{1 j}(s)\right)\right)-\tilde{f}_{j}\left(\psi_{j}\left(s-\tau_{1 j}(s)\right)\right) \tilde{h}_{j}\left(\psi_{j}\left(s-\tau_{1 j}(s)\right)\right) \mid\right) \\
& +\sum_{j=1}^{n}\left|b_{1 j}(s)\right| \int_{0}^{\infty}\left|K_{1 j}(u)\right|\left(\mid f_{j}\left(\phi_{j}(s-u)\right) h_{j}\left(\phi_{j}(s-u)\right)\right.
\end{aligned}
$$




$$
\begin{aligned}
& -f_{j}\left(\phi_{j}(s-u)\right) h_{j}\left(\psi_{j}(s-u)\right)|+| f_{j}\left(\phi_{j}(s-u)\right) h_{j}\left(\psi_{j}(s-u)\right) \\
& \left.\left.-f_{j}\left(\psi_{j}(s-u)\right) h_{j}\left(\psi_{j}(s-u)\right) \mid\right) d u\right] d s, \ldots \text {, } \\
& \int_{-\infty}^{t} e^{-\int_{s}^{t} c_{n}(u) d u}\left|\sum_{j=1}^{n}\right| a_{n j}(s) \mid\left(\mid \tilde{f}_{j}\left(\phi_{j}\left(s-\tau_{n j}(s)\right)\right) \tilde{h}_{j}\left(\phi_{j}\left(s-\tau_{n j}(s)\right)\right)\right. \\
& -\tilde{f}_{j}\left(\phi_{j}\left(s-\tau_{n j}(s)\right)\right) \tilde{h}_{j}\left(\psi_{j}\left(s-\tau_{n j}(s)\right)\right)|+| \tilde{f}_{j}\left(\phi_{j}\left(s-\tau_{n j}(s)\right)\right) \\
& \text {. } \left.\tilde{h}_{j}\left(\psi_{j}\left(s-\tau_{n j}(s)\right)\right)-\tilde{f}_{j}\left(\psi_{j}\left(s-\tau_{n j}(s)\right)\right) \tilde{h}_{j}\left(\psi_{j}\left(s-\tau_{n j}(s)\right)\right) \mid\right) \\
& +\sum_{j=1}^{n}\left|b_{n j}(s)\right| \int_{0}^{\infty}\left|K_{n j}(u)\right|\left(\mid f_{j}\left(\phi_{j}(s-u)\right) h_{j}\left(\phi_{j}(s-u)\right)-f_{j}\left(\phi_{j}(s-u)\right)\right. \\
& \cdot h_{j}\left(\psi_{j}(s-u)\right)|+| f_{j}\left(\phi_{j}(s-u)\right) h_{j}\left(\psi_{j}(s-u)\right) \\
& \left.\left.\left.-f_{j}\left(\psi_{j}(s-u)\right) h_{j}\left(\psi_{j}(s-u)\right) \mid\right) d u\right] d s\right)^{T} \\
& \leq\left(\int _ { - \infty } ^ { t } e ^ { - \int _ { s } ^ { t } c _ { 1 } ( u ) d u } \left[\left(\sum_{j=1}^{n} \overline{a_{1 j}} L_{j}^{\tilde{f}}\left(L_{j}^{\tilde{h}} \sup _{t \in R}\left|\phi_{j}(t)\right|+\tilde{M}_{j}\right)\right.\right.\right. \\
& \left.\left.+\sum_{j=1}^{n} \overline{b_{1 j}} k_{1 j} L_{j}^{f}\left(L_{j}^{h} \sup _{t \in R}\left|\phi_{j}(t)\right|+M_{j}\right)\right) \sup _{t \in R}\left|\phi_{j}(t)-\psi_{j}(t)\right|\right] d s, \ldots, \\
& \int_{-\infty}^{t} e^{-\int_{s}^{t} c_{n}(u) d u}\left[\left(\sum_{j=1}^{n} \overline{a_{n j}} L_{j}^{\tilde{f}}\left(L_{j}^{\tilde{h}} \sup _{t \in R}\left|\phi_{j}(t)\right|+\tilde{M}_{j}\right)\right.\right. \\
& \left.\left.\left.+\sum_{j=1}^{n} \overline{b_{n j}} k_{n j} L_{j}^{f}\left(L_{j}^{h} \sup _{t \in R}\left|\phi_{j}(t)\right|+M_{j}\right)\right) \sup _{t \in R}\left|\phi_{j}(t)-\psi_{j}(t)\right|\right] d s\right)^{T} \\
& \leq\left(\sum_{j=1}^{n} \tilde{c}_{1}^{-1}\left(\overline{a_{1 j}} L_{j}^{\tilde{f}}\left(L_{j}^{\tilde{h}} \frac{L}{1-\delta}+\tilde{M}_{j}\right)+\overline{b_{1 j}} k_{1 j} L_{j}^{f}\left(L_{j}^{h} \frac{L}{1-\delta}+M_{j}\right)\right)\right. \\
& \cdot \sup _{t \in R}\left|\phi_{j}(t)-\psi_{j}(t)\right|, \ldots, \sum_{j=1}^{n} \tilde{c}_{n}^{-1}\left(\overline{a_{n j}} L_{j}^{\tilde{f}}\left(L_{j}^{\tilde{h}} \frac{L}{1-\delta}+\tilde{M}_{j}\right)\right. \\
& \left.\left.+\overline{b_{n j}} k_{n j} L_{j}^{f}\left(L_{j}^{h} \frac{L}{1-\delta}+M_{j}\right)\right) \sup _{t \in R}\left|\phi_{j}(t)-\psi_{j}(t)\right|\right)^{T} \\
& \leq D\left(\sup _{t \in R}\left|\phi_{1}(t)-\psi_{1}(t)\right|, \ldots, \sup _{t \in R}\left|\phi_{n}(t)-\psi_{n}(t)\right|\right)^{T},
\end{aligned}
$$

which, together with $T^{m}$ is a self-mapping from $B^{*}$ to $B^{*}$, implies that

$$
\begin{aligned}
& \left|T^{m}(\phi(t))-T^{m}(\psi(t))\right| \\
\leq & D\left(\sup _{t \in R}\left|\left(T^{m-1}(\phi(t))-T^{m-1}(\psi(t))\right)_{1}\right|,\right. \\
& \left.\ldots, \sup _{t \in R}\left|\left(T^{m-1}(\phi(t))-T^{m-1}(\psi(t))\right)_{n}\right|\right)^{T} \\
\leq & \cdots \leq D^{m}\left(\sup _{t \in R}\left|\phi_{1}(t)-\psi_{1}(t)\right|, \ldots, \sup _{t \in R}\left|\phi_{n}(t)-\psi_{n}(t)\right|\right)^{T},
\end{aligned}
$$


where $m$ is a positive integer. Since $\rho(D)<1$, we obtain

$$
\lim _{m \longrightarrow+\infty} D^{m}=0
$$

which implies that there exist a positive integer $N$ and a positive constant $r<1$ such that

$$
D^{N}=\left(h_{i j}\right)_{n \times n}, \text { and } \sum_{j=1}^{n} h_{i j} \leq r, i=1,2, \ldots, n .
$$

In view of (2.9) and (2.10), we have

$$
\begin{aligned}
\left|\left(T^{N}(\phi(t))-T^{N}(\psi(t))\right)_{i}\right| & \leq \sup _{t \in R}\left|\left(T^{N}(\phi(t))-T^{N}(\psi(t))\right)_{i}\right| \\
& \leq \sum_{j=1}^{n} h_{i j} \sup _{t \in R}\left|\phi_{j}(t)-\psi_{j}(t)\right| \\
& \left.\leq \sup _{t \in R} \max _{1 \leq j \leq n}\left|\phi_{j}(t)-\psi_{j}(t)\right|\right) \sum_{j=1}^{n} h_{i j} \\
& \leq r\|\phi(t)-\psi(t)\|_{B},
\end{aligned}
$$

for all $t \in R, i=1,2, \ldots, n$. It follows that

$$
\begin{aligned}
\left\|T^{N}(\phi(t))-T^{N}(\psi(t))\right\|_{B} & =\sup _{t \in R} \max _{1 \leq i \leq n}\left|\left(T^{N}(\phi(t))-T^{N}(\psi(t))\right)_{i}\right| \\
& \leq r\|\phi(t)-\psi(t)\|_{B}
\end{aligned}
$$

This implies that the mapping $T^{N}: B^{*} \longrightarrow B^{*}$ is a contraction mapping. Therefore the mapping $T$ possesses a unique fixed point $Z^{*} \in B^{*}, T Z^{*}=Z^{*}$. By (2.1), $Z^{*}$ satisfies (1.1). So $Z^{*}$ is an almost periodic solution of system (1.1) in $B^{*}$. The proof of Theorem 2.1 is now complete.

\section{Uniqueness and exponential stability of the almost periodic solution}

In this section, we establish some results for the uniqueness and exponential stability of the almost periodic solution of (1.1).

Theorem 3.1. Let $\left(H_{4}\right)$ hold. Suppose that all the conditions of Theorem 2.1 are satisfied. Then system (1.1) has exactly one almost periodic solution $Z^{*}(t)$. Moreover, $Z^{*}(t)$ is globally exponentially stable.

Proof. From Theorem 2.1, system (1.1) has at least one almost periodic solution $Z^{*}(t)=\left\{x_{j}^{*}(t)\right\}$ with initial value $\varphi^{*}=\left\{\varphi_{j}^{*}(t)\right\}$, and $Z^{*}(t) \in B^{*}$. Let $Z(t)=$ $\left\{x_{j}(t)\right\}$ be an arbitrary solution of system (1.1) with initial value $\varphi=\left\{\varphi_{j}(t)\right\}$, let $y(t)=\left\{y_{j}(t)\right\}=\left\{x_{j}(t)-x_{j}^{*}(t)\right\}=Z(t)-Z^{*}(t)$. Then

$$
y_{i}^{\prime}(t)=-c_{i}(t) y_{i}(t)
$$




$$
\begin{aligned}
& +\sum_{j=1}^{n} a_{i j}(t)\left(\tilde{g}_{j}\left(y_{j}\left(t-\tau_{i j}(t)\right)+x_{j}^{*}\left(t-\tau_{i j}(t)\right)\right)-\tilde{g}_{j}\left(x_{j}^{*}\left(t-\tau_{i j}(t)\right)\right)\right) \\
& +\sum_{j=1}^{n} b_{i j}(t) \int_{0}^{\infty} K_{i j}(u)\left(g_{j}\left(y_{j}(t-u)+x_{j}^{*}(t-u)\right)-g_{j}\left(x_{j}^{*}(t-u)\right)\right) d u
\end{aligned}
$$

where $i=1,2, \ldots, n$.

Since $\rho(D)<1$, it follows from Lemma 1.4 that $E_{n}-D$ is an $M$-matrix. In view of Lemma 1.3 , there exists a constant vector $\bar{\xi}=\left(\bar{\xi}_{1}, \bar{\xi}_{2}, \ldots, \bar{\xi}_{n}\right)^{T}>$ $(0,0, \ldots, 0)^{T}$ such that

$$
\left(E_{n}-D\right) \vec{\xi}>(0,0, \ldots, 0)^{T}
$$

Then,

$$
-\tilde{c}_{i} \bar{\xi}_{i}+\sum_{j=1}^{n} \overline{a_{i j}} L_{j}^{\tilde{f}}\left(\tilde{M}_{j}+\frac{L}{1-\delta} L_{j}^{\tilde{h}}\right) \bar{\xi}_{j}+\sum_{j=1}^{n} \widetilde{b_{i j}} k_{i j} L_{j}^{f}\left(M_{j}+\frac{L}{1-\delta} L_{j}^{h}\right) \bar{\xi}_{j}<0
$$

for $i=1,2, \ldots, n$. Therefore, we can choose a constant $d>1$ such that

$$
\begin{aligned}
\xi_{i} & =d \bar{\xi}_{i}>\sup _{-\infty<t \leq 0}\left|y_{i}(t)\right| \\
& =\sup _{-\infty \leq s \leq 0} \max _{1 \leq i \leq n}\left|\varphi_{i}(s)-\varphi_{i}^{*}(s)\right|, \quad i=1,2, \ldots, n,
\end{aligned}
$$

and

$$
\begin{aligned}
& -\tilde{c}_{i} \xi_{i}+\sum_{j=1}^{n} \overline{a_{i j}} L_{j}^{\tilde{f}}\left(\tilde{M}_{j}+\frac{L}{1-\delta} L_{j}^{\tilde{h}}\right) \xi_{j}+\sum_{j=1}^{n} \overline{b_{i j}} k_{i j} L_{j}^{f}\left(M_{j}+\frac{L}{1-\delta} L_{j}^{h}\right) \xi_{j} \\
= & d\left[-\tilde{c}_{i} \bar{\xi}_{i}+\sum_{j=1}^{n} \overline{a_{i j}} L_{j}^{\tilde{f}}\left(\tilde{M}_{j}+\frac{L}{1-\delta} L_{j}^{\tilde{h}}\right) \bar{\xi}_{j}+\sum_{j=1}^{n} \overline{b_{i j}} k_{i j} L_{j}^{f}\left(M_{j}+\frac{L}{1-\delta} L_{j}^{h}\right) \bar{\xi}_{j}\right] \\
< & 0, i=1,2, \ldots, n .
\end{aligned}
$$

Set

$$
\begin{aligned}
\Gamma_{i}(\omega)= & \left(\omega-\tilde{c}_{i}\right) \xi_{i}+\sum_{j=1}^{n} \overline{a_{i j}} L_{j}^{\tilde{f}}\left(\tilde{M}_{j}+\frac{L}{1-\delta} L_{j}^{\tilde{h}}\right) \xi_{j} e^{\omega \tau} \\
& +\sum_{j=1}^{n} \overline{b_{i j}} L_{j}^{f}\left(M_{j}+\frac{L}{1-\delta} L_{j}^{h}\right) \xi_{j} \int_{0}^{\infty}\left|K_{i j}(u)\right| e^{\omega u} d u
\end{aligned}
$$

where $i=1,2, \ldots, n$. Clearly, $\Gamma_{i}(\omega), i=1,2, \ldots, n$, are continuous functions on $\left[0, \lambda_{0}\right]$. Since

$$
\Gamma_{i}(0)=-\tilde{c}_{i} \xi_{i}+\sum_{j=1}^{n} \widehat{a_{i j}} L_{j}^{\tilde{f}}\left(\tilde{M}_{j}+\frac{L}{1-\delta} L_{j}^{\tilde{h}}\right) \xi_{j}
$$




$$
\begin{aligned}
& +\sum_{j=1}^{n} \overline{b_{i j}} L_{j}^{f}\left(M_{j}+\frac{L}{1-\delta} L_{j}^{h}\right) \xi_{j} \int_{0}^{\infty}\left|K_{i j}(u)\right| d u \\
\leq & -\tilde{c}_{i} \xi_{i}+\sum_{j=1}^{n} \overline{a_{i j}} L_{j}^{\tilde{f}}\left(\tilde{M}_{j}+\frac{L}{1-\delta} L_{j}^{\tilde{h}}\right) \xi_{j} \\
& +\sum_{j=1}^{n} \overline{b_{i j}} k_{i j} L_{j}^{f}\left(M_{j}+\frac{L}{1-\delta} L_{j}^{h}\right) \xi_{j}<0,
\end{aligned}
$$

where $i=1,2, \ldots, n$, we can choose a positive constant $\lambda \in\left[0, \lambda_{0}\right]$ such that (3.6)

$$
\begin{aligned}
\Gamma_{i}(\lambda)= & \left(\lambda-\tilde{c}_{i}\right) \xi_{i}+\sum_{j=1}^{n} \overline{a_{i j}} L_{j}^{\tilde{f}}\left(\tilde{M}_{j}+\frac{L}{1-\delta} L_{j}^{\tilde{h}}\right) \xi_{j} e^{\lambda \tau} \\
& +\sum_{j=1}^{n} \overline{b_{i j}} L_{j}^{f}\left(M_{j}+\frac{L}{1-\delta} L_{j}^{h}\right) \xi_{j} \int_{0}^{\infty}\left|K_{i j}(u)\right| e^{\lambda u} d u<0, i=1,2, \ldots, n .
\end{aligned}
$$

We consider the Lyapunov functional

$$
V_{i}(t)=\left|y_{i}(t)\right| e^{\lambda t}, \quad i=1,2, \ldots, n .
$$

Calculating the upper right derivative of $V_{i}(t)$ along the solution $y(t)=\left\{y_{j}(t)\right\}$ of system (3.1) with the initial value $\bar{\varphi}=\varphi-\varphi^{*}$, from $(2.5),(2.6),(3.1)$ and $\left(H_{1}\right)$, we have

$$
\begin{aligned}
& D^{+}\left(V_{i}(t)\right) \\
\leq & -c_{i}(t)\left|y_{i}(t)\right| e^{\lambda t}+\sum_{j=1}^{n} \mid a_{i j}(t)\left(\tilde{g}_{j}\left(y_{j}\left(t-\tau_{i j}(t)\right)+x_{j}^{*}\left(t-\tau_{i j}(t)\right)\right)\right. \\
& \left.-\tilde{g}_{j}\left(x_{j}^{*}\left(t-\tau_{i j}(t)\right)\right)\right)\left|e^{\lambda t}+\sum_{j=1}^{n}\right| b_{i j}(t) \int_{0}^{\infty} K_{i j}(u)\left(g_{j}\left(y_{j}(t-u)+x_{j}^{*}(t-u)\right)\right. \\
& \left.-g_{j}\left(x_{j}^{*}(t-u)\right)\right) d u\left|e^{\lambda t}+\lambda\right| y_{i}(t) \mid e^{\lambda t} \\
\leq & -\tilde{c}_{i}\left|y_{i}(t)\right| e^{\lambda t}+\sum_{j=1}^{n}\left[| a _ { i j } ( t ) | \left(\mid \tilde{f}_{j}\left(y_{j}\left(t-\tau_{i j}(t)\right)+x_{j}^{*}\left(t-\tau_{i j}(t)\right)\right) \tilde{h}_{j}\left(y_{j}\left(t-\tau_{i j}(t)\right)\right.\right.\right. \\
& \left.+x_{j}^{*}\left(t-\tau_{i j}(t)\right)\right)-\tilde{f}_{j}\left(x_{j}^{*}\left(t-\tau_{i j}(t)\right)\right) \tilde{h}_{j}\left(y_{j}\left(t-\tau_{i j}(t)\right)+x_{j}^{*}\left(t-\tau_{i j}(t)\right)\right) \mid \\
& +\mid \tilde{f}_{j}\left(x_{j}^{*}\left(t-\tau_{i j}(t)\right)\right) \tilde{h}_{j}\left(y_{j}\left(t-\tau_{i j}(t)\right)+x_{j}^{*}\left(t-\tau_{i j}(t)\right)\right)-\tilde{f}_{j}\left(x_{j}^{*}\left(t-\tau_{i j}(t)\right)\right) \\
& +\tilde{h}_{j}\left(x_{j}^{*}\left(t-\tau_{i j}(t)\right)\right) \mid e^{\lambda t} \\
& +\sum_{j=1}^{n}\left[| b _ { i j } ( t ) | \int _ { 0 } ^ { \infty } | K _ { i j } ( u ) | \left(\mid f_{j}\left(y_{j}(t-u)+x_{j}^{*}(t-u)\right)\right.\right.
\end{aligned}
$$




$$
\begin{aligned}
& \cdot h_{j}\left(y_{j}(t-u)+x_{j}^{*}(t-u)\right)-f_{j}\left(x_{j}^{*}(t-u)\right) h_{j}\left(y_{j}(t-u)+x_{j}^{*}(t-u)\right) \mid \\
& +\left|f_{j}\left(x_{j}^{*}(t-u)\right) h_{j}\left(y_{j}(t-u)+x_{j}^{*}(t-u)\right)-f_{j}\left(x_{j}^{*}(t-u)\right) h_{j}\left(x_{j}^{*}(t-u)\right)\right| d u e^{\lambda t} \\
& +\lambda\left|y_{i}(t)\right| e^{\lambda t} \\
\leq & \left(\lambda-\tilde{c}_{i}\right)\left|y_{i}(t)\right| e^{\lambda t}+\sum_{j=1}^{n} \overline{a_{i j}} L_{j}^{\tilde{f}}\left(\tilde{M}_{j}+\frac{L}{1-\delta} L_{j}^{\tilde{h}}\right)\left|y_{j}\left(t-\tau_{i j}(t)\right)\right| e^{\lambda t} \\
& +\sum_{j=1}^{n} \overline{b_{i j}} L_{j}^{f}\left(M_{j}+\frac{L}{1-\delta} L_{j}^{h}\right) \int_{0}^{\infty}\left|K_{i j}(u)\right|\left|y_{j}(t-u)\right| d u e^{\lambda t},
\end{aligned}
$$

where $i=1,2, \ldots, n$.

We claim that

$$
V_{i}(t)=\left|y_{i}(t)\right| e^{\lambda t}<\xi_{i} \quad \text { for all } t>0, \quad i=1,2, \ldots, n .
$$

Contrarily, there must exist $i \in\{1,2, \ldots, n\}$ and $t_{i}>0$ such that

$$
V_{i}\left(t_{i}\right)=\xi_{i} \text { and } V_{j}(t)<\xi_{3}, \forall t \in\left(-\infty, t_{i}\right), j=1,2, \ldots, n,
$$

which implies that

(3.11) $V_{i}\left(t_{i}\right)-\xi_{i}=0$ and $V_{j}(t)-\xi_{j}<0, \forall t \in\left(-\infty, t_{i}\right), j=1,2, \ldots, n$.

Together with (3.8) and (3.11), we obtain

$$
\begin{aligned}
0 \leq & D^{+}\left(V_{i}\left(t_{i}\right)-\xi_{i}\right) \\
= & D^{+}\left(V_{i}\left(t_{i}\right)\right) \\
\leq & \left(\lambda-\tilde{c}_{i}\right)\left|y_{i}\left(t_{i}\right)\right| e^{\lambda t_{i}}+\sum_{j=1}^{n} \overline{a_{i j}} L_{j}^{\tilde{f}}\left(\tilde{M}_{j}+\frac{L}{1-\delta} L_{j}^{\tilde{h}}\right)\left|y_{j}\left(t_{i}-\tau_{i j}\left(t_{i}\right)\right)\right| e^{\lambda t_{i}} \\
& +\sum_{j=1}^{n} \overline{b_{i j}} L_{j}^{f}\left(M_{j}+\frac{L}{1-\delta} L_{j}^{h}\right) \int_{0}^{\infty}\left|K_{i j}(u)\right|\left|y_{j}\left(t_{i}-u\right)\right| d u e^{\lambda t_{i}} \\
= & \left(\lambda-\tilde{c}_{i}\right)\left|y_{i}\left(t_{i}\right)\right| e^{\lambda t_{i}} \\
& +\sum_{j=1}^{n} \widehat{a_{i j}} L_{j}^{f}\left(\tilde{M}_{j}+\frac{L}{1-\delta} L_{j}^{\tilde{h}}\right)\left|y_{j}\left(t_{i}-\tau_{i j}\left(t_{i}\right)\right)\right| e^{\lambda\left(t_{i}-\tau_{i j}\left(t_{i}\right)\right)} e^{\lambda \tau_{i j}\left(t_{i}\right)} \\
& +\sum_{j=1}^{n} \widehat{b_{i j}} L_{j}^{f}\left(M_{j}+\frac{L}{1-\delta} L_{j}^{h}\right) \int_{0}^{\infty}\left|K_{i j}(u)\right|\left|y_{j}\left(t_{i}-u\right)\right| e^{\lambda\left(t_{i}-u\right)} e^{\lambda u} d u \\
\leq & \left(\lambda-\tilde{c}_{i}\right) \xi_{i}+\sum_{j=1}^{n} \overline{a_{i j}} L_{j}^{\tilde{f}}\left(\tilde{M}_{j}+\frac{L^{\prime}}{1-\delta} L_{j}^{\tilde{h}}\right) \xi_{j} e^{\lambda \tau} \\
& +\sum_{j=1}^{n} \widehat{b_{i j}} L_{j}^{f}\left(M_{j}+\frac{L}{1-\delta} L_{j}^{h}\right) \xi_{j} \int_{0}^{\infty}\left|K_{i j}(u)\right| e^{\lambda u} d u .
\end{aligned}
$$


Thus,

$$
\begin{aligned}
0 \leq & \left(\lambda-\tilde{c}_{i}\right) \xi_{i}+\sum_{j=1}^{n} \overline{a_{i j}} L_{j}^{\tilde{f}}\left(\tilde{M}_{j}+\frac{L}{1-\delta} L_{j}^{\tilde{h}}\right) \xi_{j} e^{\lambda \tau} \\
& +\sum_{j=1}^{n} \overline{b_{i j}} L_{j}^{f}\left(M_{j}+\frac{L}{1-\delta} L_{j}^{h}\right) \xi_{j} \int_{0}^{\infty}\left|K_{i j}(u)\right| e^{\lambda u} d u,
\end{aligned}
$$

which contradicts (3.6). Hence, (3.9) holds.

Letting $\|\bar{\varphi}\|=\left\|\varphi-\varphi^{*}\right\|>0$, we can choose a constant $M_{\varphi}>1$ such that

$$
\max _{1 \leq i \leq n}\left\{\xi_{i}\right\} \leq M_{\varphi}\left\|\varphi-\varphi^{*}\right\|, \quad i=1,2, \ldots, n
$$

In view of (3.12) and (3.13), we get

$$
\left|x_{i}(t)-x_{i}^{*}(t)\right|=\left|y_{i}(t)\right| \leq \max _{1 \leq i \leq n}\left\{\xi_{i}\right\} e^{-\lambda t} \leq M_{\varphi}\left\|\varphi-\varphi^{*}\right\| e^{-\lambda t},
$$

where $i=1,2, \ldots, n, t>0$. This completes the proof.

\section{An example}

In this section, we give an example to demonstrate the results obtained in previous sections.

Example 4.1. Consider the following CNNs with delays:

$$
\left\{\begin{aligned}
x_{1}^{\prime}(t)= & -c_{1}(t) x_{1}(t)+\frac{1}{2}(\sin t) \tilde{g}_{1}\left(x_{1}\left(t-\sin ^{2} t\right)\right) \\
& +\frac{1}{2}(\cos t) \tilde{g}_{2}\left(x_{2}\left(t-2 \sin ^{2} t\right)\right)+\frac{1}{2}(\sin t) \int_{0}^{\infty} e^{-u} g_{1}\left(x_{1}(t-u)\right) d u \\
& +\frac{1}{2}(\cos t) \int_{0}^{\infty} e^{-u} g_{2}\left(x_{2}(t-u)\right) d u+\frac{3}{4} \sin (\sqrt{2} t) \\
x_{2}^{\prime}(t)= & -c_{2}(t) x_{2}(t)+\frac{1}{2}(\sin 2 t) g_{1}\left(x_{1}\left(t-3 \cos ^{2} t\right)\right) \\
& +\frac{1}{2}(\cos 4 t) g_{2}\left(x_{2}\left(t-4 \sin ^{2} t\right)\right)+\frac{1}{2}(\sin 2 t) \int_{0}^{\infty} e^{-u} g_{1}\left(x_{1}(t-u)\right) d u \\
& +\frac{1}{2}(\cos 4 t) \int_{0}^{\infty} e^{-u} g_{2}\left(x_{2}(t-u)\right) d u+\frac{3}{4} \cos (\sqrt{2} t),
\end{aligned}\right.
$$

where $c_{1}(t)=1+\sin ^{2}(\sqrt{3} t), c_{2}(t)=1+\sin ^{4}(\sqrt{5} t), \tilde{g}_{i}(x)=g_{i}(x)=\frac{1}{8}|x| \sin x$. Observe that $\tilde{f}_{i}(x)=f_{i}(x)=\frac{1}{8}|x|, \tilde{h}_{i}(x)=h_{i}(x)=\sin x, \tilde{c}_{1}=\tilde{c}_{2}=\tilde{M}_{i}=$ $M_{i}=L_{i}^{\tilde{h}}=L_{i}^{h}=1, \quad L_{i}^{\tilde{f}}=L_{i}^{f}=\frac{1}{8}, \overline{a_{i j}}=\overline{b_{i j}}=\frac{1}{2}, k_{i j}=1, i, j=1,2$. Then

$$
\begin{gathered}
L=\frac{3}{4}, \quad \delta=\frac{1}{4}<1, \\
\|D\|_{1}=\max _{1 \leq i \leq 2}\left\{\sum_{j=1}^{2} \tilde{c}_{i}^{-1}\left(\overline{a_{i j}} L_{j}^{\tilde{f}}\left(L_{j}^{\tilde{h}} \frac{L}{1-\delta}+\tilde{M}_{j}\right)+\overline{b_{i j}} k_{i j} L_{j}^{f}\left(L_{j}^{h} \frac{L}{1-\delta}+M_{j}\right)\right)\right\} \\
=\frac{1}{2}<1,
\end{gathered}
$$

where $\|\cdot\|_{1}$ is the row norm of matrix. It is straight forward to check that all the conditions needed in Theorem 3.1 are satisfied. Therefore, by Theorem 3.1, system (4.1) has exactly one almost periodic solution, which is globally exponentially stable. 
Remark 4.1. System (4.1) is a very simple form of CNNs. One can observe that $\tilde{g}_{1}(x)=\tilde{g}_{2}(x)=g_{1}(x)=g_{2}(x)=\frac{1}{8}|x| \sin x$ and the condition $\left(H_{0}\right)$ is not satisfied. Therefore, all the results in [1-11] and the references cited therein can not be applicable to system (4.1). This implies that the results of this paper are essentially new.

\section{Conclusions}

In this paper, cellular neural networks with time-varying delays and continuously distributed delays have been studied. Without assuming the global Lipschitz conditions of activation functions, some sufficient conditions for the existence and exponential stability of the almost periodic solutions have been established. These obtained results are new and they complement previously known results. Moreover, an example is given to illustrate the effectiveness of our results

\section{References}

[1] A. Berman and R. J. Plemmons, Nonnegative Matrices in the Mathematical Science, Academic Press, New York, 1979.

[2] J. Cao, Global exponential stability and periodic solutions of delayed cellular neural networks, J. Comput. System Sci. 60 (2000), no. 1, 38-46.

[3] _ New results concerning exponential stability and periodic solutions of delayed cellular neural networks, Phys. Lett. A 307 (2003), no. 2-3, 136-147.

[4] A. Chen and J. Cao, Existence and attractivity of almost periodic solutions for cellular neural networks with distributed delays and variable coefficients, Appl. Math. Comput. 134 (2003), no. 1, 125-140.

[5] A. Chen and L. H. Huang, Existence and attractivity of almost periodic solutions of Hopfield neural networks, Acta Math. Sci. Ser. A Chin. Ed. 21 (2001), no. 4, 505-511.

[6] Q. Dong, K. Matsui, and X. Huang, Existence and stability of periodic solutions for Hopfield neural network equations with periodic input, Nonlinear Anal. 49 (2002), no. 4, Ser. A: Theory Methods, 471-479.

[7] A. M. Fink, Almost periodic differential equations, Lecture Notes in Mathematics, Vol. 377. Springer-Verlag, Berlin-New York, 1974.

[8] J. Hale and S. M. Verduyn Lunel, Introduction to functional-differential equations, Applied Mathematical Sciences, 99. Springer-Verlag, New York, 1993.

[9] C. Y. He, Almost periodic differential equation, Higher Education Publishing House, Beijing, 1992.

[10] X. Huang and J. Cao, Almost periodic solution of shunting inhibitory cellular neural networks with time-varying delay, Phys. Lett. A 314 (2003), no. 3, 222-231.

[11] H. Huang, J. Cao, and J. Wang, Global exponential stability and periodic solutions of recurrent neural networks with delays, Phys. Lett. A 298 (2002), no. 5-6, 393-404.

[12] B. Liu and L. Huang, Existence and exponential stability of almost periodic solutions for cellular neural networks with time-varying delays, Phys. Lett. A 341 (2005), 135-144.

[13] __ Existence and exponential stability of almost periodic solutions for Hopfield neural networks with delays, Neurocomputing 68 (2005), 196-207.

[14] Z. Liu, A. Chen, J. Cao, and L. Huang, Existence and global exponential stability of almost periodic solutions of BAM neural networks with continuously distributed delays, Phys. Lett. A 319 (2003), no. 3-4, 305-316. 
[15] Z. Liu and L. Liao, Existence and global exponential stability of periodic solution of cellular neural networks with time-varying delays, J. Math. Anal. Appl. 290 (2004), no. 1, 247-262.

DEPARTMENT OF Mathematics

Hunan University of ARTs and ScIence Changde

415000 P. R. China

E-mail address: 1iubw007@yahoo.com.cn 